\title{
Form factors in relativistic quantum mechanic: constraints from space-time translations
}

\section{Bertrand DESPLANQUES*}

Laboratoire de Physique Subatomique et Cosmologie, Université Joseph Fourier Grenoble 1, CNRS/IN2P3, INPG, France

E-mail: desplanqelpsc.in2p3.fr

\section{Yu-bing DONG}

Institute of High Energy Physics, Chinese Academy of Science,

Beijing 100049, P. R. China

E-mail: dongyb@mail.ihep.ac.cn

The comparison of form factors calculated from a single-particle current in different relativistic quantum mechanic approaches evidences tremendous discrepancies. The role of constraints coming from space-time translations is considered here with this respect. It is known that invariance under these translations implies the energy-momentum conservation relation that is usually assumed to hold globally. Transformations of the current under these translations, which lead to this result, also imply constraints that have been ignored so far in relativistic quantum mechanic approaches. An implementation of these constraints is discussed in the case of a model with two scalar constituents. It amounts to incorporate selected two-body currents to all orders in the interaction. Discrepancies for form factors in different approaches can thus be removed, contributing to restore the equivalence of different approaches. Results for the standard front-form approach $\left(q^{+}=0\right)$ are found to fulfill the constraints and are therefore unchanged. The relation with results from a dispersion-relation approach is also made.

LIGHT CONE 2008 Relativistic Nuclear and Particle Physics

July 7-11, 2008

Mulhouse, France

\footnotetext{
${ }^{*}$ Speaker.
} 


\section{Introduction}

Examination of form factors calculated in different forms of relativistic quantum mechanic (RQM) [1,2], with the same solution of a mass operator, shows tremendous differences depending on the approach that is used [3,4]. On the other hand, fitting the wave function to experiment shows that it depends strongly on the approach $[5,6]$. At first sight, one cannot therefore decide whether some discrepancy is due to the chosen implementation of relativity or to the underlying dynamics. It is however believed that results should not depend on the choice of the hypersurface underlying some form of relativity [7] and that their equivalence requires the introduction of manybody currents.

Among properties expected from Poincaré covariance, invariance of form factors under rotations or boosts can easily be checked by applying these transformations to the system under consideration. In absence of a similar test, the role of space-time translations, which are also part of the Poincare group, is much less known beyond the energy-momentum conservation that is, of course, assumed to hold. In this contribution, we examine this role and show that accounting for the constraints they imply can remove large discrepancies between results obtained in different approaches with the simplest one-body current.

In the following, we successively consider the space-time translations and constraints they imply (sect. 2), the implementation of these constraints (sect. 3), the illustration of their role for a pion-like system (sect. 4) and, finally, the relation to a dispersion-relation approach that could be considered as the convergence point of all approaches based on the same solution of a mass operator (sect. 5). Due to lack of space, details had to be skipped. They could be found in ref. [8].

\section{Constraints from Poincaré space-time translation invariance}

Under space-time translations, a vector or a scalar current transforms as follows:

$$
e^{i P \cdot a} J^{v}(x)(\text { or } S(x)) e^{-i P \cdot a}=J^{v}(x+a)(\text { or } S(x+a)),
$$

where $P^{\mu}$ represents the total momentum operator. Considering the matrix element of this equality between eigenstates of $P^{\mu}$ for $a=-x$, one gets:

$$
<i \mid J^{v}(x)(\text { or } S(x))\left|f>=e^{i\left(P_{i}-P_{f}\right) \cdot x}<i\right| J^{v}(0)(\text { or } S(0)) \mid f>.
$$

Together with a field carrying momentum $q^{\mu}$, one gets under the assumption of space-time translation invariance the current momentum-energy conservation, $\left(P_{f}-P_{i}\right)^{\mu}=q^{\mu}$.

For the purpose of calculating form factors, $J^{v}(0)$ (or $S(0)$ ) is generally approximated by a one-body current. Equation (2.1) however implies further relations involving the commutator of $P^{\mu}$ with the currents and the derivative of the current with respect to $x$ [9]. Particularly interesting relations are the following double commutators:

$$
\left[P_{\mu},\left[P^{\mu}, J^{v}(x)\right]\right]=-\partial_{\mu} \partial^{\mu} J^{v}(x),\left[P_{\mu},\left[P^{\mu}, S(x)\right]\right]=-\partial_{\mu} \partial^{\mu} S(x) .
$$

Considering the matrix element of these relations between eigenstates of $P^{\mu}$, and for the case of a one-body current, one should verify the relation:

$$
<\mid q^{2} J^{v}(0)(\text { or } S(0))|>=<|\left(p_{i}-p_{f}\right)^{2} J^{v}(0)(\text { or } S(0)) \mid>\text {. }
$$


It is easily seen that this equation cannot be generally fulfilled, as most often $q^{2} \neq\left(p_{i}-p_{f}\right)^{2}$ in RQM approaches (see fig. 1 for a graphical representation). This implies that the assumption of a single-particle current is inconsistent with properties from space-time translations and that the current, $J^{v}(0)$ (or $S(0)$ ), besides a one-body component, should also contain many-body components which, until now, have been ignored.

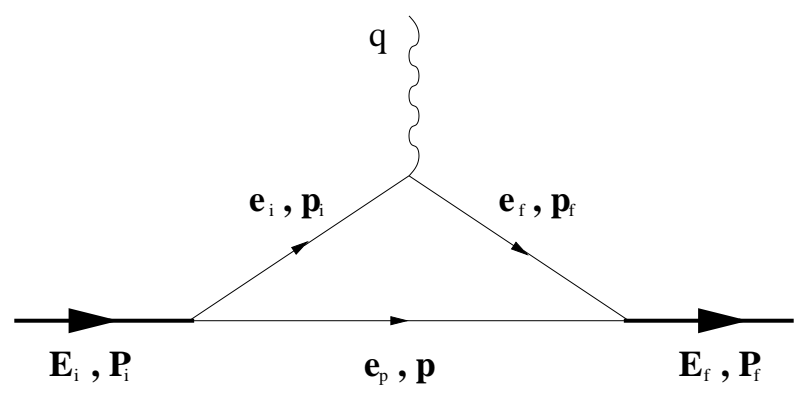

Figure 1: Representation of the interaction with an external probe

\section{Implementation of the constraints}

It is expected that many-body currents at all orders in the interaction are required to fulfill constraints from space-time translations. As considering these currents explicitly is excluded, we consider them indirectly, by modifying wave functions and the current operator. The modification is suggested by examining expressions of form factors in various approaches, which show that the factor multiplying $Q$ could be given by a factor varying from 1 in some cases to $\frac{2 e_{k}}{M}$ in other ones [10]. The departure of this quantity to 1 represents an interaction term and is a signature of the hypersurface underlying the approach. To account for the constraints, we thus propose to multiply $Q$ by a factor $\alpha$ and determine this one by requiring that the squared momentum transferred to the system, $q^{2}$, be equal to the one for the constituents, denoted " $\left(p_{i}-p_{f}\right)$ "2. The equation to be solved is typically given by:

$$
\begin{aligned}
q^{2} & ="\left[\left(P_{i}-P_{f}\right)^{2}+2\left(\Delta_{i}-\Delta_{f}\right)\left(P_{i}-P_{f}\right) \cdot \xi+\left(\Delta_{i}-\Delta_{f}\right)^{2} \xi^{2}\right] " \\
& =\alpha^{2} q^{2}-2 \alpha "\left(\Delta_{i}-\Delta_{f}\right) " q \cdot \xi+"\left(\Delta_{i}-\Delta_{f}\right) " \xi^{2},
\end{aligned}
$$

where $\Delta$, which represents an interaction effect, also depends on $\alpha$. Explicit expressions of $\alpha$ can be found in ref. [8] for different forms. Expressions for form factors, taking into account the effect of constraints motivated by space-time translation properties, can then be obtained [8]. By expanding these expressions in terms of $\Delta$, the many-body character of corrections at all orders of the interaction could be checked.

A few points deserve to be noticed. Firstly, for the standard front-form where $\xi^{2}=0, q$. $\xi$ (or $\left.q^{+}\right)=0$, the factor $\alpha$ is equal to 1 and, therefore, results for the form factors are unchanged. Secondly, a solution has been found but we do not exclude that the implementation can be done differently, what would be desirable to fulfill the infinite set of constraints involving the multiple commutators of $P^{\mu}$ with currents. Thirdly, the choice of the charge current is constrained for some part. We used for our purpose a current inspired from results for the simplest Feynman triangle diagram. This allows one to get Lorentz invariant results for form factors. 


\section{Numerical illustration}
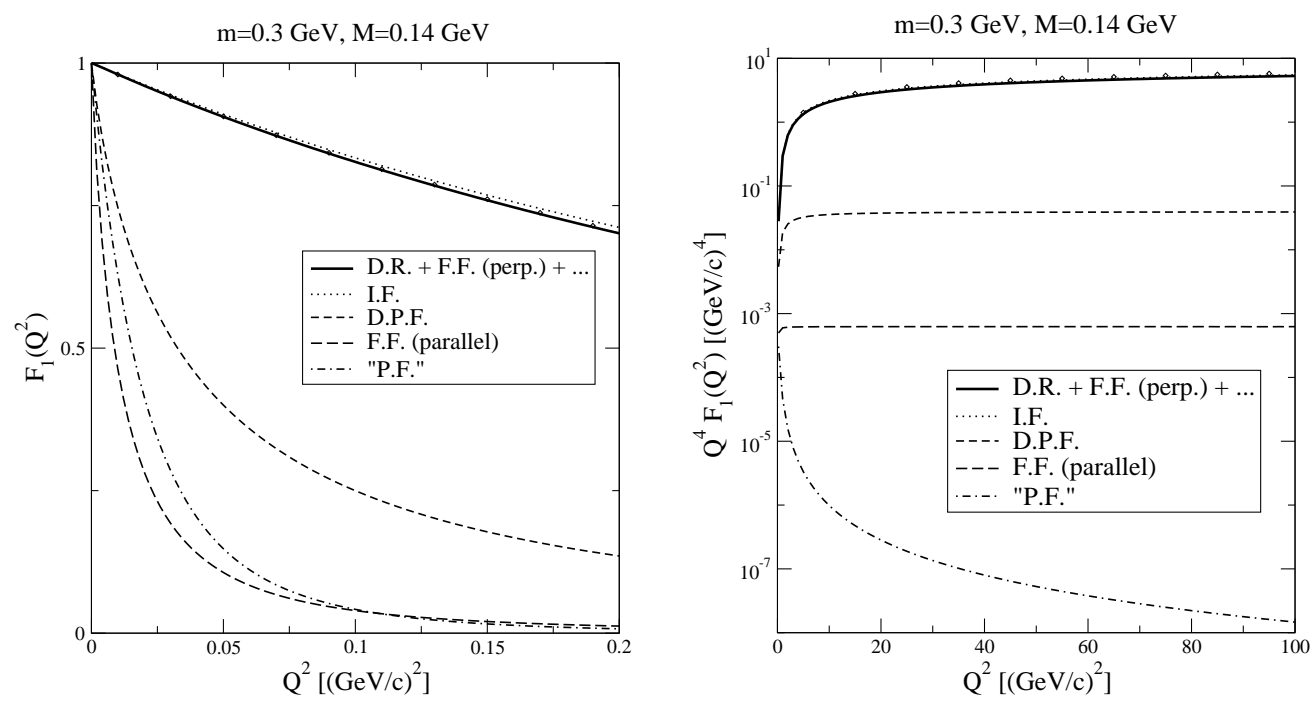

Figure 2: Charge form factors. See text for the curves. The "experiment" is represented by diamonds.

To illustrate effects of the restoration of properties related to space-time translations, we consider a system of scalar particles interacting through the exchange of massless particles (WickCutkosky model [11, 12]). For this system, that may be used as a test case [13], form factors can be calculated exactly. There are two of them for the ground state, a charge and a scalar one, which provide us with some "experiment". The RQM calculations are based on the solution of a mass operator where the interaction is chosen so that to reproduce approximately the degeneracy pattern of the exact spectrum while keeping the high-momentum power law unchanged. The strength is fitted to reproduce the energy of the ground state used in the calculations. The mass of the system and of the constituents are those currently used for the pion, $M=0.14 \mathrm{GeV}$ and $m=0.3 \mathrm{GeV}$.
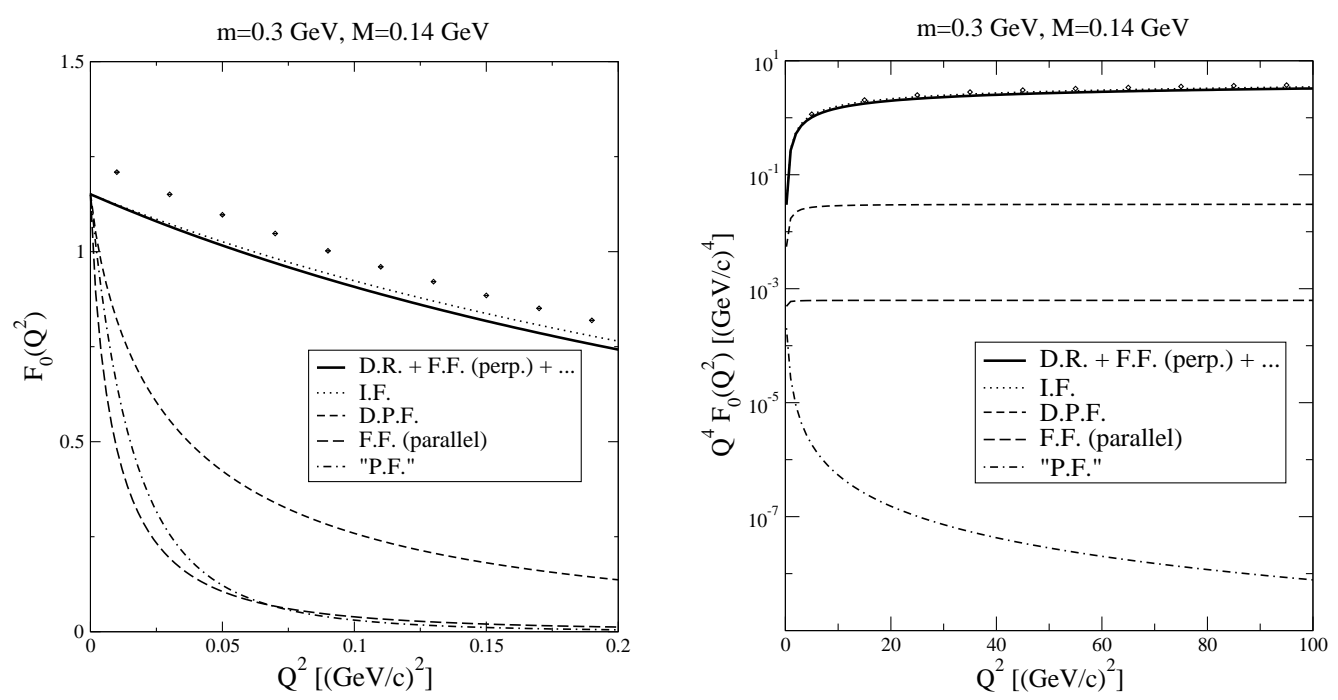

Figure 3: Scalar form factors. See text for the curves. The "experiment" is represented by diamonds. 
Results are presented in figs. 2, 3 for the charge and scalar form factors. In each case, there are two panels describing the low- and high- $Q^{2}$ behavior, respectively sensitive to the square radius and to the asymptotic behavior. The different curves correspond to uncorrected Breit-frame form factors for the front form $\left(q^{+}=0\right.$; F.F. (perp.)), a front form with a parallel configuration (F.F. (parallel)), the usual instant form (I.F.) [14], some instant form with the symmetry properties of the point form ("P.F.") [15], a point form inspired from the Dirac one (D.P.F.) [16], and corrected results which, all, coincide with the standard front-form results $\left(q^{+}=0\right)$ that are unchanged. Anticipating on next section, we also show results of a dispersion-relation approach (D.R.), which coincide with the standard front-form ones.

Examination of results for the charge form factor (fig. 2) shows that tremendous discrepancies are removed by accounting for constraints from space-time translation properties at both low and high $Q^{2}$. The paradox of a charge radius tending to infinity while the mass of the system goes to zero (or the interaction is increased) disappears. There remain a slight discrepancy with "experiment". It can be ascribed for a part to the description of the mass operator. It is not clear whether genuine two-body currents are needed. Examination of results for the scalar form factor (fig. 3) shows similar features as far as corrections related to space-time translation properties are concerned. In contrast to the charge form factor, genuine two-body currents are needed here to explain the form factor at low $Q^{2}$. It is reminded that, contrary to the charge form factor, the scalar one at $Q^{2}=0$ is not protected by some conservation law.

\section{Relation to a dispersion-relation approach}

A dispersion-relation has been proposed to calculate form factors [17, 18, 19]. The expressions for the charge and scalar form factors read:

$$
\begin{aligned}
F_{1}\left(Q^{2}\right) & =\frac{1}{N} \iint d \bar{s} d\left(\frac{s_{i}-s_{f}}{Q}\right) \frac{\left(2 \bar{s}+Q^{2}\right) \theta\left(\frac{s_{i} s_{f}}{D}-m^{2}\right)}{D^{3 / 2}} \phi\left(s_{f}\right) \phi\left(s_{i}\right), \\
F_{0}\left(Q^{2}\right) & =\frac{1}{N} \iint d \bar{s} d\left(\frac{s_{i}-s_{f}}{Q}\right) \frac{\theta\left(\frac{s_{i} s_{f}}{D}-m^{2}\right)}{2 D^{1 / 2}} \phi\left(s_{f}\right) \phi\left(s_{i}\right), \\
\text { where } N & =\int d s \sqrt{\frac{s-4 m^{2}}{s}} \phi^{2}(s), \quad \bar{s}=\frac{s_{i}+s_{f}}{2}, \quad D=4 \bar{s}+Q^{2}+\frac{\left(s_{i}-s_{f}\right)^{2}}{Q^{2}} .
\end{aligned}
$$

This approach was presented as an instant-form one [18]. In absence of reference to a particular direction, it could be thought as a point-form one. Moreover, one of the authors (B.D.) found that the above expressions could be obtained from the standard front-form ones $\left(q^{+}=0\right)$ by an appropriate change of variables. A similar result was obtained independently by Melikhov [19] for the pion case. Examining the approach, it is noticed that it is based on the free scattering amplitude in an external field. The interaction effects that are here or there in RQM approaches are therefore absent. The variables $s_{i}=\left(p_{i}+p\right)^{2}, s_{f}=\left(p_{f}+p\right)^{2}$ and $\left(p_{i}-p_{f}\right)^{2}$ refer to on-mass shell constituents. The functions $\phi(s)=\tilde{\phi}\left(k^{2}=\frac{s}{4}-m^{2}\right)$ can therefore be identified to the solution of a mass operator used in RQM approaches. The relation $q^{2}=\left(p_{i}-p_{f}\right)^{2}$, which ensures that the square momentum transferred to the system be equal to the one transferred to the constituents, fulfills the constraints expected from space-time translation properties. These three features suggest that the RQM results 
for form factors should converge to the dispersion-relation ones, once the above constraints are accounted for.

As the relations of corrected RQM form factors to the dispersion-relation ones are not well known, we give here expressions evidencing the relation for the charge form factor. The explicit form of the change of variables can be found in ref. [8]. The first case refers to the standard front form approach $\left(q^{+}=0\right)$, which is of particular interest for this light-cone meeting. Using the $k_{\perp}$ and $x$ variables currently employed in this domain, the relation reads:

$$
\begin{aligned}
F_{1}\left(Q^{2}\right) & =\frac{2}{\pi N} \int \frac{d^{2} p_{\perp} d x}{2 x(1-x)} \tilde{\phi}\left(\vec{k}_{f}^{2}\right) \tilde{\phi}\left(\vec{k}_{i}^{2}\right) \\
& =\frac{2}{\pi N} \iint d \bar{s} d\left(\frac{s_{i}-s_{f}}{Q}\right) \frac{1}{2 \sqrt{D}} \phi\left(s_{f}\right) \phi\left(s_{i}\right) \times \int \frac{d x\left(\left(2 \bar{s}+Q^{2}\right) / D-(x-d)\right)}{\sqrt{\left(\frac{s_{i} s_{f}}{D}-m^{2}\right) f-(x-d)^{2}}} \\
& =\frac{1}{N} \iint d \bar{s} d\left(\frac{s_{i}-s_{f}}{Q}\right) \frac{\left(2 \bar{s}+Q^{2}\right) \theta(\cdots)}{D^{3 / 2}} \phi\left(s_{f}\right) \phi\left(s_{i}\right)
\end{aligned}
$$

The second case refers to an arbitrary front orientation $\lambda^{\mu}$ with finite $\lambda^{2}$. The relation reads:

$$
\begin{aligned}
F_{1}\left(Q^{2}\right) & =\frac{16 \pi^{2}}{N(2 \pi)^{3}} \int \frac{d \vec{p}}{e_{p}} \frac{“\left(2 p+p_{i}+p_{f}\right) " \cdot \lambda}{2 “\left(p_{i}+p_{f}\right) " \cdot \lambda} " \tilde{\phi}\left(\vec{k}_{f}^{2}\right) \tilde{\phi}\left(\vec{k}_{i}^{2}\right) ” \\
& =\frac{2}{\pi N} \iint d \bar{s} d\left(\frac{s_{i}-s_{f}}{Q}\right) \frac{\theta(\cdots)}{4 D^{3 / 2}} \phi\left(s_{f}\right) \phi\left(s_{i}\right) \times \sum \int \frac{d(p \cdot \hat{\lambda})\left(\left(2 \bar{s}+Q^{2}\right)-(p \cdot \hat{\lambda}-d) g\right)}{\sqrt{\left(\frac{s_{i} s_{f}}{D}-m^{2}\right) f-(p \cdot \hat{\lambda}-d)^{2}}} .
\end{aligned}
$$

In this case, it is noticed that the dependence on $\lambda^{\mu}$ and $\bar{P}^{\mu}=\left(P_{i}+P_{f}\right)^{\mu} / 2$, which characterizes quantities $d, f$ and $g$ [8], disappears in the integration over $p \cdot \hat{\lambda}$, allowing one to get Lorentzinvariant results. In both cases, the integral is reduced from a three- to a two-dimensional one.

\section{Conclusion}

In this contribution, we mainly considered properties related to Poincaré space-time translations in RQM approaches for the calculation of form factors. The current practice is to use these properties to factorize the $x$ dependence of the current, allowing one to get the usual energymomentum conservation relation, and to assume that the current at $x=0$ is a one-body current. At first sight, there is no relation of the 4-momentum transferred to the system and to the constituents as in a field-theory approach. Considering further relations implied by the transformation of the current under space-time translations, we showed that they could not be satisfied with a current reduced to a one-body component, pointing to the necessary presence of many-body components. These ones allow us to account in RQM approaches for the equality of the momentum transferred to the whole system and to the constituents, which characterizes a field-theory approach. We described a method to implement the above many-body contributions in the case of a scalar-particle model. When this is done, it is found that discrepancies between different RQM approaches for calculating form factors can be removed, showing that the role of space-time translations extends 
beyond the standard energy-momentum conservation. It is also found that these results could coincide with those of a dispersion-relation approach. Altogether, all aspects of the Poincaré group (rotations, boosts and space-time translations) are essential in getting reliable estimates of form factors as far as the implementation of relativity is concerned. Possible discrepancies with experiment can thus be more likely ascribed to the underlying dynamics.

Present results can be extended without much difficulty to elastic as well as inelastic form factors of 0 -spin systems consisting of 1/2-spin constituents with unequal masses. Extension to non-zero spin systems could require more elaboration.

\section{Acknowledgments}

One of the authors (B.D.) is very grateful to V.A. Karmanov and W. Polyzou for an initiation to implementation of relativity in field theory and relativistic quantum mechanic respectively. It is likely that the complementary knowledge of these two approaches was essential in obtaining results presented here. This work is partly supported by the National Sciences Foundations of China under grant No. 10775148.

\section{References}

[1] P.A.M. Dirac, Rev. Mod. Phys. 21 (1949) 392.

[2] B. Keister, W. Polyzou, Adv. Nucl. Phys. 20 (1991) 225.

[3] A. Amghar, B. Desplanques, L. Theuß1, Nucl. Phys. A 714 (2003) 213.

[4] B. Desplanques, [nucl-th/0407074].

[5] Jun He, B. Julia-Diaz, Yu-bing Dong, Phys. Lett. B 602 (2004) 212.

[6] B. Julia-Diaz, D.O. Riska, F. Coester, Phys. Rev. C 69 (2004) 035212, Erratum Phys. Rev. C 75 (2007) 069902.

[7] S.N. Sokolov, A.N. Shatnii, Theor. Math. Phys. 37 (1978) 1029.

[8] B. Desplanques, Yu-bing Dong, Eur. Phys. J. A 37 (2008) 33.

[9] F.M. Lev, Rivista del Nuovo Cimento 16 (1993) 1.

[10] B. Desplanques, L. Theuß1, Eur. Phys. J. A 21 (2004) 93.

[11] G.C. Wick, Phys. Rev. 96 (1954) 1124

[12] R.E. Cutkosky, Phys. Rev. 96 (1954) 1135.

[13] V.A. Karmanov, A.V. Smirnov, Nucl. Phys. A 546 (1992) 691.

[14] B. Bakamjian, L.H. Thomas, Phys. Rev. 92 (1953) 1300.

[15] B. Bakamjian, Phys. Rev. 121 (1961) 1849.

[16] B. Desplanques, Nucl. Phys. A 748 (2005) 139.

[17] V.V. Anisovich et al., Nucl. Phys. A 544 (1992) 747.

[18] A.F. Krutov, V.E. Troitsky, Phys. Rev. C 65 (2002) 045501.

[19] D. Melikhov, Eur. Phys. J. direct C4 (2002) 2 [hep-ph/0110087]. 